\title{
A model for disorder in fluorine-intercalated graphite
}

\author{
S. L. di Vittorio \\ Department of Materials Science and Engineering. Massachuse'ts Institute of Technology, \\ Cambridge, Massachusetts 02139 \\ M. S. Dresselhaus \\ Department of Electrical Engineering and Computer Science and Deparment of Physics. \\ Massachusetts Institute of Technology, Cambridge, Massachusetts 02139 \\ G. Dresselhaus \\ Francis Bitter National Magnet Laboratory, Massachusetts Institute of Technology, \\ Cambridge. Massachusetts 02139
}

(Received 27 July 1992: accepted 18 March 1993)

The structural and electronic propertics of fluorine- and brominc-intercalated graphite fibers and HOPG are summarized. In contrast to the bromine intercalate, which is purely ionic for any experimentally attainable intercalate concentration, fluorine has a dual ionic and covalent behavior in graphitc. Furthermore, whereas bromine-intercalated graphite is ordered, fluorine-intercalated graphite is disordered. The stiff graphene planes are buckled and islands of various fluorine concentrations are formed. A thermodynamic model is proposed that accounts for the differences between fluorine- and bromine-intercalated graphite materials. The model describes the competition between ionically bonded and covalently bonded intercalate phases of fluorine in graphite. Covalent bonding is more favorable energetically, but an important nucleation barrier exists due to strain and to the destruction of the conjugation of the double bonds.

\section{INTRODUCTION}

Transport measurements, ${ }^{1.3}$ transmission electron microscopy (TEM), ${ }^{4}$ electron spin resonance (ESR), ${ }^{5}$ and optical ${ }^{\circ}$ measurements have established the presence of disorder in fluorine graphite intercalation compounds (GIC's) and have helped characterize the nature of this disorder, both structurally and electronically. It is now clear that the propertics and structure of fluorine GIC's are quite different from that of most other known GIC's. Usually the driving force for intercalation is the formation of an ionic bond between the intercalated species and the carricr-poor graphene planes. The charge transfer increases the metallic character of the intercalation compound as compared to pristine graphite. In contrast, most fluorine GIC's are more resistive than pristine graphite, and beyond a concentration of $\mathrm{C}_{4} \mathrm{~F}$, they exhibit a semiconducting dependence of resistivity on temperature. ${ }^{3}$ For another thing, the metallic bonding of the intercalate to the carbon atoms (through the $p_{z}$ orbitals) is much too weak to disturb the very strong $s p^{2}$ in-plane carbon-carbon bonding, ${ }^{8}$ so that the very stiff and planar graphene sheets remain essentially undisturbed by the intercalation process. In contrast, this planarity of the carbon planes does not persist after intercalation of fluorine, and TEM micrographs show that the carbon planes in fluorine GIC's are buckled. ${ }^{4}$

The unique electronic and structural properties of fluorine GIC's are especially striking when compared to other halogen GIC's, which have a similar chemical structure. The purpose of this paper is thus to explain the differences between fluorine GIC's and other GIC's, with special reference to halogen GIC's. In this connection, we will apply our model more specifically to fluorine and bromine GIC's.

Indeed, not all halogen atoms or molecules can be intercalated into graphitc. As a matter of fact, only bromine, fluorine, and the compounds $\mathrm{ICl}$ and $\mathrm{IBr}$ do intercalate into graphite. Chlorine intercalates with much difficulty; the reaction takes place only at low reaction temperatures and the kinetics are exceedingly slow. ${ }^{9}$ Iodine does not intercalate. This wide range of behaviors among the halogen atoms can be explained by the interplay of the intramolecular bonding energy $E_{X-X}$ and the intramolecular distance $d_{X-X}$ of the $X_{2}$ molecule, where $X$ stands for a halogen atom. ${ }^{7}$ Indeed, $\mathrm{X}_{2}$ molecules have a minimum in encrgy when $d_{X-X}$ is commensurate with the host graphite lattice, i.c., when the separation between the two halogen atoms is as close as possible to the distance between the centers of the two adjacent graphite hexagons, namely $2.46 \AA$. In the case of fluorine, the intramolecular bond of the $F_{2}$ molecule is weak, so that it is cnergetically more favorable to have as the guest species fluorine atoms rather than a heavily distorted molecule. It is likely that molecular fluorine enters the lattice and becomes atomic either after intercalation or through the intercalation process itself. Bromine intercalates as a molecule because the 
bromine-bromine distance, $d_{\mathrm{Br}-\mathrm{Br}}=2.41 \AA$, is close to $2.46 \AA$. The other halogen molecules do not intercalate because it is both costly energetically to break the molecules apart and because the intramolecular distances are very different from $2.46 \AA$. This hypothesis is confirmed by the fact that the two molecules $\mathrm{IBr}$ and $\mathrm{ICl}$, with intramolecular distances close to $2.46 \AA$, do intercalate. Table I summarizes the intramolecular distances and energies for the various halogen molecules. The data are extracted from Landoldt-Börnstein. ${ }^{10}$

In Sec. II, we first summarize the structure and electronic properties of F-GIC's and X-GIC's. In Sec. III, we go on to present the different types of bonds that can be formed between carbon and intercalated halogen atoms. We proceed to present a thermodynamic model that explains the structural differences between F-GIC's and X-GIC's in Sec. IV. This model is then applied to a variety of experimental behaviors of fluorine GIC's in Sec. $\mathrm{V}$.

\section{NATURE OF THE DISORDER}

\section{A. Fluorine}

From a structural point of vicw, the superlattice structure of the fluorine GIC's can be clearly observed by TEM, although the interlayer repeat distance $I_{c}$ is not sharp but rather is spread over a distribution of values. ${ }^{4}$ The width of the $I_{c}$ value distribution increases as the fluorine concentration increases. The $C_{x} F$ GIC's are found to exhibit good staging behavior for stage I to stage IV samples (no $\mathrm{C}_{x} \mathrm{~F}$ samples of higher stage have been experimentally synthesized). ${ }^{11}$ However, a given stage does not correspond to a specific fluorine concentration. Rather, samples with a wide range of fluorine concentrations may have the same stage. For example, $\mathrm{C}_{x} \mathrm{~F}$ samples with $4.5 \geqslant x \geqslant 2.9$ all exhibit the stage I structure.

The distinctive feature of the graphenc planes in fluorine GIC's, as compared to other acceptor or donor GIC's, is the presence of disorder, characterized by the nonplanar nature of the graphene planes. ${ }^{4}$ In addition to this waviness, the fluorine GIC's are inhomogeneous, ${ }^{4}$ forming islands about $70 \AA$ in diameter, with varying interlayer repeat distances $I_{c}$, corresponding to varying fluorine concentrations. No substantial nonintercalated

TABI.E I. The intramolecular distance $d_{X-X}$ and binding energy $E_{X} \cdot X$ for various halogen molccules.

\begin{tabular}{lcccccc}
\hline \multicolumn{1}{c}{ Species X } & $\mathrm{I}$ & $\mathrm{Br}$ & $\mathrm{Cl}$ & $\mathrm{F}$ & $\mathrm{IBr}$ & $\mathrm{ICl}$ \\
\hline$d_{X-X}(\AA)$ & & & & & & \\
$E_{X-X}(\mathrm{kcal} / \mathrm{mole})$ & 35.6 & 2.41 & 2.02 & 1.42 & 2.49 & 2.40 \\
$\mathrm{GIC}^{\mathrm{N}}$ & $\mathrm{No}$ & $\mathrm{Br}_{2}$ & $\mathrm{No}$ & $\mathrm{F}$ & $\mathrm{IBr}$ & $\mathrm{ICl}$ \\
\hline
\end{tabular}

The listing gives the halogen specics that can be intercalated. regions can be seen by TEM in the most concentrated samples, consistent with $\mathrm{x}$-ray diffraction measurements on the stage I samples, where no (002) diffraction peak is present. Despite the presence of disorder, the anisotropy in the conductivity of fluorine GIC's, $\sigma_{a} / \sigma_{c}>10^{5}$ (where $\sigma_{a}$ and $\sigma_{c}$ are the in-plane and out-of-plane conductivities, respectively) remains extremely high. ${ }^{12}$

No long-range order is present in the fluorinc layer, whose structure is that of a two-dimensional random solid at room temperature. It is emphasized here that the chemical nature of the fluorine intercalate in graphite is atomic, as opposed to other halogens which retain their molccular nature upon intercalation. A bump in the resistivity curve around $130 \mathrm{~K}-180 \mathrm{~K}$ suggests the possibility of an in-plane fluorine ordering transition, but such a transition has not been established conclusively and remains hypothetical. ${ }^{13}$

Electronically, fluorine in graphite has a mixed ionic and covalent behavior. ${ }^{14}$ For dilute fluorine concentrations, fluorine GIC's are found to be ionic with a charge transfer of $1 / 6$ of a hole per intercalated fluorine atom in the limit of vanishing fluorine concentration. ${ }^{6}$ However, as the fluorine concentration increases, the bonding behavior of fluorine shifts to covalent bonding, with a sharp decrease in the carrier concentration. "Thus, the $\mathrm{C}_{x} \mathrm{~F}$ GIC's contain both conduction electrons and localized spins at the Fermi level. Usually, disordered materials have either conduction electrons or localized spins at the Fermi level, depending on the location of the mobility edge with respect to the Fermi level. ${ }^{5}$ In $\mathrm{C}_{x}$ F GIC's, because of the inhomogeneity, concentrated islands with localized spins coexist along with dilute islands with conduction electron spins. As the average fluorine concentration increases, the density of localized spins increases, but a substantial concentration of conduction electron spins is still present in the material.

\section{B. Bromine}

In contrast, bromine forms an ionic acceptor graphite intercalation compound, with a charge transfer of approximately $0.2-0.3$ holes per intercalated brominc atom. ${ }^{7}$ It is believed that this partial charge transfer does not vary with intercalated bromine concentration nor does it vary with staging, ${ }^{7}$ though, in our opinion, these points are not well established. As a result of the charge transfer, bromine GIC's exhibit electronic properties characteristic of a metal, such as a positive temperature cocfficient of the resistivity, ${ }^{15}$ Shubnikov-de-Haas oscillations, Pauli susceptibility, etc.

The most concentrated sample that can be achicved by bromine intercalation is the stage Il $\mathrm{C}_{16} \mathrm{Br}_{2} \mathrm{com}$ pound. ${ }^{7}$ Only a few discrete bromine concentrations $\mathrm{C}_{16} \mathrm{Br}_{2}, \mathrm{C}_{24} \mathrm{Br}_{2}, \mathrm{C}_{32} \mathrm{Br}_{2}$, and $\mathrm{C}_{40} \mathrm{Br}_{2}$, corresponding to stages II, III, IV, and $\mathrm{V}$, respectively, can be experi- 
mentally achieved. Although difficulty in synthesizing stage I samples is common in GIC's, the reason for the absence of stage I bromine GIC is not well understood. It is not related to the large size of bromine, since stage I ICl GIC's, which are comparable in size, can be synthesized. More likely, it is related to the interaction energy between neighboring bromine intercalate layers. Structurally, bromine GIC's exhibit straight lattice fringes; i.e., the process of bromine intercalation hardly disturbs the graphene planes. ${ }^{16}$

The staging behaviors of the fluorine and bromine arc easily explained by the respective random incommensurate and ordered commensurate structures of the intercalate layers. Because the $\mathrm{Br}-\mathrm{Br}$ distance is fixed, an increase in bromine concentration can be achieved only by changing the staging so that stages and intercalate concentrations closcly match cach other. On the other hand, because of the random location of the fluorine intercalate, higher fluorine concentrations can be achieved by a closer packing of the fluorine atoms in the fluorine plane, thus leading to a range of fluorine concentrations corresponding to the same nominal stage.

\section{BONDING IN GRAPHITE}

When halogen atoms or molecules are intercalated into graphite, two possible kinds of bonds can be formed between the carbon and the halogen atoms: ionic bonds and covalent bonds. The nature of the fluorine-carbon ionic bond is very similar to the nature of the bromine-carbon ionic bond; a similar partial charge of $1 / 6$ of a hole is transferred to the graphene planes for each ionic bond. ${ }^{6,7}$ The driving force for this transfer is the high electronegativity of bromine $(F=3)$ and fluorine $(F=4)$ as compared to graphite. Since the ionic bonding involves the $p_{z}$ orbitals of the carbon that extend perpendicular to the graphene planes, the ionic bonding does not disturb the planar nature of the graphene planes.

On the other hand, covalent bonding disturbs the $s p^{2}$ bonding of the hexagonal network. Because of the strong $s p^{2}$ bonding of graphite, strong oxidants such as the halogen atoms are needed to form a covalent bond. The carbon atoms of pristine graphite are in the $s p^{2}$ hybridization and form $120^{\circ}$ angles between two bonds at each carbon atom vertex. After covalent bonding with a halogen atom, the carbon atom is in an $s p^{3}$ hybridization state, with bonds to three carbon atoms and one halogen atom. The $s p^{3}$ bonded carbon reaches its minimum in energy when the four bonds form a regular tetrahedron, i.e., for an angle of $109^{\circ} 28^{\prime}$. Since the minimum in encrgy of the neighboring carbons requires $120^{\circ}$ angles between bonds, covalent bonding of a carbon to a halogen introduces a large strain into the planar structure. The carbon atom is pushed out of the graphene plane, with the angles to its three carbon neighbors intermediate between $109^{\circ}$ and $120^{\circ}$.

Finally, graphite, similar to benzene, is normally described as a structure resonating between two electron distributions, as shown in Fig. 1. This phenomenon, which is called conjugation, or resonance, physically corresponds to an equal delocalization of the electrons on the six carbons of the ring. Conjugation lowers the energy of the molecule (by $6 \mathrm{kcal} / \mathrm{mole}$ of carbon in the case of benzene) and thus stabilizes the benzene structure. ${ }^{17}$ After addition of a halogen molecule to the benzene ring, this resonance is partially destroyed since the alternation of double bonds around the ring disappears. The electrons are only delocalized over four carbon atoms, similar to butadiene $(0.6 \mathrm{kcal} / \mathrm{mole}$ of carbon), as shown in Fig. 1. The resonance of the benzene structure accounts for the ease of substituting bromine for hydrogen in benzene, and the extreme difficulty for brominc additions on a double bond. ${ }^{17}$ With respect to double bond conjugation, graphite is very similar to benzene, but instead of being delocalized over a ring, the carriers are delocalized over all the graphene planes. Therefore, the conjugation energy of graphite is large.

\section{A MODEL FOR FLUORINE GIC'S}

In this simple model, the total energy of the halogenintercalated graphite compound with stoichiometry

\section{Benzene}<smiles>c1ccccc1</smiles>

Resonance energy $=6 \mathrm{kcal} / \mathrm{mole}$

After halogen $x$ addition

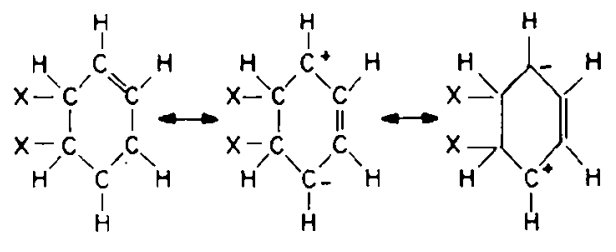

Resonance energy $=0.6 \mathrm{kcol} /$ mole some os butadiene

FIG. 1. The two resonant structures of benzene which give rise to a resonance energy of $6 \mathrm{kcal} / \mathrm{mole}$ of carbon. After addition of one and two halogen atoms $X$, the resonance energy $E_{\text {res }}$ is reduced to that of pentadiene and butadiene, $0.6 \mathrm{kcal} / \mathrm{mole}$ of carbon. 
$\mathrm{CF}_{y}$ is

$$
\begin{aligned}
E(f, y) \approx & (1-f) y E_{\mathrm{ion}}+f y E_{\mathrm{cov}} \\
& +E_{\mathrm{strain}}(f, y)+E_{\mathrm{res}}(f, y)
\end{aligned}
$$

where $f$ is the fraction of covalent bonds, and $E_{\mathrm{ion}}, E_{\mathrm{cov}}$, $E_{\text {strain }}$, and $E_{\text {res }}$ are the energies of the ionic bond and the covalent bond, the strain encrgy and the resonance energy, respectively. The model takes into account the difference in energy between $\mathrm{C}-\mathrm{X}$ ionic and covalent bonds, the strain caused by covalent bonds in the middle of an ionic matrix, and the destruction of double bond conjugation. As for this latter conjugation, an important difference between benzene and graphite needs to be pointed out. In the case of bromine, two atoms are simultaneously added on the double bond of benzene bccause addition of a single bromine atom leads to a highly unstable structure with a dangling bond. However, in the case of graphite, additions of either one or two halogen atoms lead to highly strained and energetically unfavorable structures. Therefore, the addition of a single halogen atom in graphite cannot be ruled out and will be further discussed in relationship to the nucleation of the covalent phase.

It should be stated here that the model of Eq. 1 is oversimplified because it neglects other important energy contributions, most notably the interaction between neighboring intercalate atoms, and the presence of defects in the pristine graphite materials. Additionally, other less significant factors such as entropic contributions, which lead to the order-disorder transition in the bromine layer at high temperature, ${ }^{18}$ or the interaction between neighboring intercalate layers are neglected. We will see below, however, that this simple model explains a large number of experimental facts about fluorine GIC's, such as the transition from ionic bonding to covalent bonding, the island behavior, and why the more disordered pristine carbon materials are more covalently bonded than the highly graphitic materials for the same fluorine intercalate concentration. The model also explains why bromine bonding is purely ionic, whereas fluorine bonding has a mixed ionic and covalent character, which was the purpose of constructing the model in the first place.

Let us now give some estimates for the energies of each term involved in Eq. (1). Formation of a covalent $\mathrm{C}-\mathrm{X}$ bond releases the enormous bonding energy of $E_{\mathrm{C}-\mathrm{F}}=-114 \mathrm{kcal} / \mathrm{mole}$ in the case of fluorine, and the lesser bonding energy $E_{\mathrm{C}-\mathrm{Br}}=-65 \mathrm{kcal} / \mathrm{mole}$ in the case of bromine. When halogen bonds covalently to carbon, the carbon-carbon bond is changed from $s p^{2}$ to $s p^{3}$. Since the $s p^{2} \mathrm{C}-\mathrm{C}$ bonding energy $E_{s p^{2}}=$ $-103 \mathrm{kcal} / \mathrm{mole}$ is larger than the $s p^{3} \mathrm{C}-\mathrm{C}$ bonding energy $E_{s p^{3}}=-83 \mathrm{kcal} / \mathrm{mole}$, the actual energy gain for covalent bonding is

$$
E_{\text {total }}=E_{\mathrm{C}-\mathrm{X}}+3\left(E_{s p^{3}}-E_{s p^{2}}\right) .
$$

Equation (2) was reached by considering that when a halogen atom bonds covalently to carbon, the three neighboring carbon-carbon bonds are transformed from $s p^{2}$ bonds into $s p^{3}$ bonds. As a result, formation of a covalent carbon-fluorine bond releases an energy $E_{\text {cov }}=$ $-54 \mathrm{kcal} / \mathrm{mole}$ for fluorine, and $E_{\mathrm{cov}}=-5 \mathrm{kcal} / \mathrm{mole}$ for bromine. The carbon-halogen ionic bond energy $E_{\text {ion }}$ can be cstimated using the electronic affinities of fluorine $(3.4 \mathrm{cV})$ and bromine $(3.36 \mathrm{cV})$, and a partial charge transfer of $1 / 6$ of a hole per ionic bond. The calculation yields an energy $E_{\mathrm{ion}}=-13 \mathrm{kcal} / \mathrm{molc}$ for both fluorine and bromine ionic bonds. The energies of the various carbon-carbon and carbon-halogen bonds used in this model are summarized in Table II.

As mentioned above, the resonance energy of graphite is $E_{\text {res }}=-6 \mathrm{kcal} / \mathrm{mole}$. Finally, the strain energy can be estimated bascd on the bonding cnergy of the $\mathrm{C}-\mathrm{C} s p^{3}$ bonds. If both carbon atoms had $s p^{3}$ orbitals pointing toward each other, the $s p^{3}$ bonding energy would be $-83 \mathrm{kcal} / \mathrm{molc}$. Actually, one carbon has $s p^{3}$ orbitals and the other one has $s p^{2}$ orbitals, which significantly reduces the overlap of the orbitals of the two carbon atoms, which is described in terms of strain cnergy. We estimate the strain energy to be a fraction of the bonding energy, i.c., several tens of $\mathrm{kcal} / \mathrm{mole}$. A more accurate value for $E_{\text {strain }}$ would require a detailed calculation of the overlap of the $s p^{2}$ and $s p^{3}$ orbitals, taking into account first- and possibly second-neighbor carbon atoms. Indecd, the deformation is not limited to the carbon atom that bonds covalently with the halogen, but is also accommodated by its carbon neighbors. At any rate, the model is not sensitive to the accuracy of the valuc used for the strain encrgy.

We now discuss qualitatively the dependence of $E_{\text {strain }}(f, y)$ and $E_{\text {res }}(f, y)$ on the halogen concentration. In so doing, we will limit our analysis to the range $0 \leqslant y \leqslant 0.5$ in $\mathrm{CF}_{y}$. As mentioned above, GIC samples with intercalate concentrations up to $\mathrm{C}_{2.9} \mathrm{~F}$ or up to $\mathrm{C}_{8.0} \mathrm{Br}$ have been synthesized experimentally. Here $\mathrm{C}$ corresponds to graphite, whereas $\mathrm{C}_{2} \mathrm{~F}(y=0.5)$ is a black layered covalent solid, where all carbon-carbon

TABLE II. The bonding energies for various bonds used in the model.

\begin{tabular}{cc}
\hline Bond & Bond energy (kcal/mole) \\
\hline$s p^{2} \mathrm{C}-\mathrm{C}$ & 103 \\
$s p^{3} \mathrm{C}-\mathrm{C}$ & 83 \\
Covalent C-F & 114 \\
Ionic C-F & 13 \\
Covalent C-Br & 65 \\
Ionic C-Br & 13 \\
\hline
\end{tabular}


and carbon-fluorine bonds are $s p^{3}$ bonds. For the sake of completeness, let us mention here the existence of another fluorine-based organic covalent solid, with formula $\mathrm{CF}^{2}$, which is also a layered material containing only $s p^{3}$ bonds. Since the purpose of this paper is the study of the transition from an ionically bonded solid to a covalently bonded solid, either $\mathrm{CF}$ or $\mathrm{CF}_{2}$ could be chosen as the final point of our transition. In this respect, the structure of $\mathrm{CF}$ is closer to that of the dilute GIC's, since all carbon-carbon bonds are in-plane bonds, whereas in $\mathrm{CF}_{2}$, some interlayer carbon-carbon bonds are formed. It will be shown below that the large disorder makes it impossible to ever obtain a crystalline layered solid that is completely covalent by increasing the fluorine concentration of the GIC's. The endpoint of the reaction (CF or $\left(\mathrm{CF}_{2}\right.$ ) chosen here is thus of no particular importance.

In the limit of a dilute concentration of covalent bonds, each covalent bond creates a localized strain, and this is cncrgetically costly. The total strain encrgy increases with increasing covalent bonding until the point is reached where the numbers of $s p^{3}$ and $s p^{2}$ bonds are equal, which corresponds to a maximum in strain energy. At this point, all carbon-carbon bonds are strained. In terms of stoichiometry, this maximum in energy probably corresponds to a ratio of four carbons to one covalently bonded halogen. (Fxperimentally, the $\mathrm{C}_{4} \mathrm{~F}$ compound is still mostly an ionic solid. ${ }^{2}$ ) When the number of $s p^{3}$ bonds exceeds the number of $s p^{2}$ bonds, the $s p^{2}$ bonds can be considered to cause the strain. The strain then decreases with increasing covalent bonding from the halogens, making it highly favorable energetically to form more covalent bonds. The strain energy as a function of covalent halogen concentration is sketched in Fig. 2.

The resonance energy $E_{\text {res }}(f, y)$ (which is negative for graphite corresponding to a stabilization) decreases stecply in magnitude with increasing $s p^{3}$ bonding and vanishes when approximately every third carbon atom is covalently bonded to a halogen atom. This trend is shown in Fig. 2. It should be emphasized that the very high resonance energy of graphite, similar to benzene and other aromatic molecules, is related to the presence of a cyclical ring structure. Indeed, the resonance energy for benzene is $39 \mathrm{kcal} / \mathrm{mole}$, whereas it is in the range of a few $\mathrm{kcal} / \mathrm{mole}$ for noncyclic molecules, such as butadiene or pentadiene. Therefore, as soon as the formation of one covalent bond breaks the alternation of single and double bonds, the resonance energy almost entirely vanishes. From Fig. 2, one can sec that the dependence of the strain energy and the resonance energy on the density of covalent fluorine-carbon bonds is highly nonlinear. This nonlinearity creates an cncrgy barrier for covalent bonding of halogens, which we are now going to discuss. Before going further, we
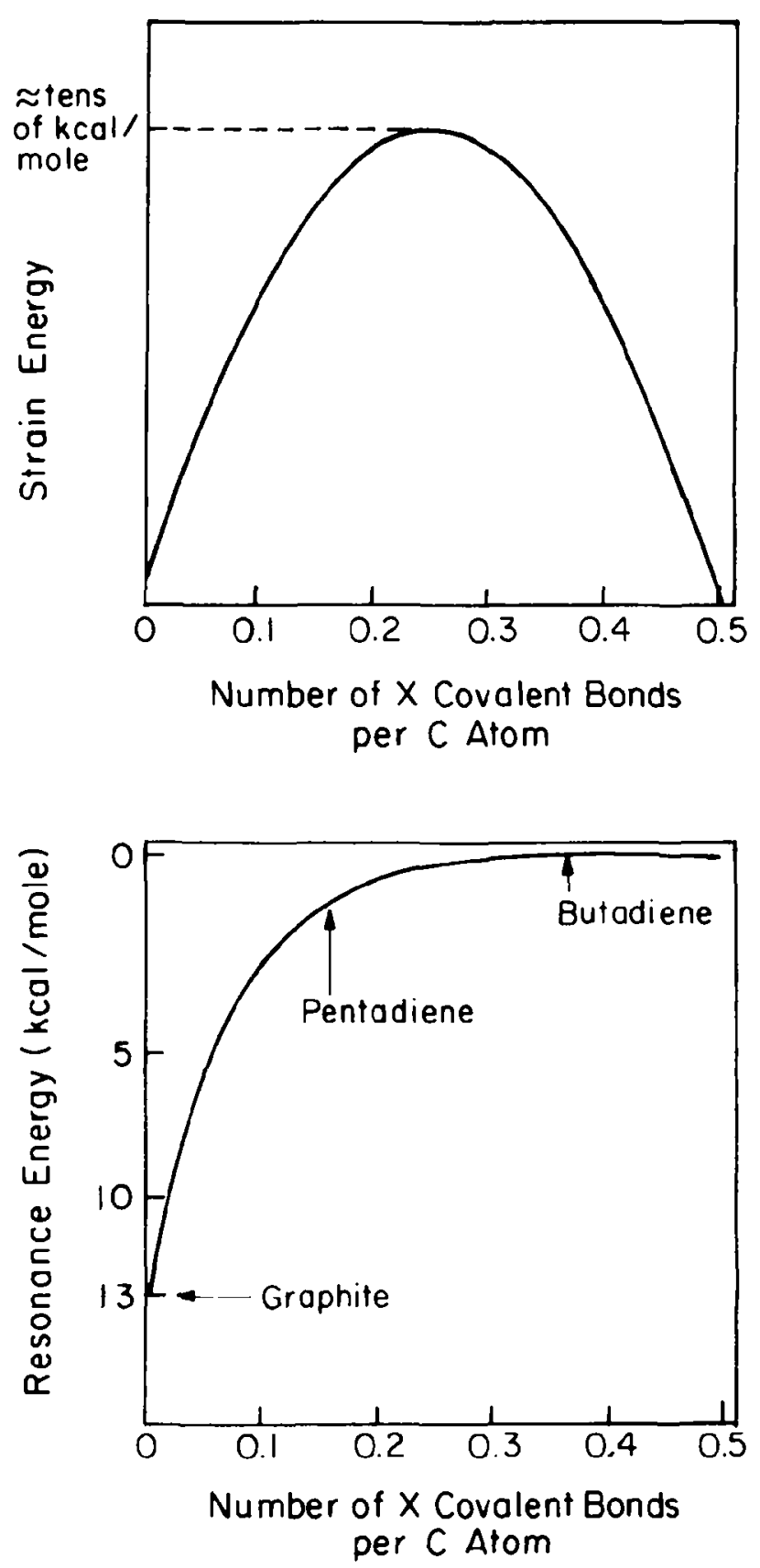

FIG. 2. A schematic of the dependence of the strain energy and the resonance energy per mole of carbon on the number of covalent halogen bonds per carbon atom.

want to emphasize that both strain and resonance energies are functions of the covalent halogen concentration only, and do not depend on the ionic halogen concentration.

The dependence of $E(f, y)$ on the intercalated halogen concentration in the two cases of ionic bonds and covalent bonds is shown in Fig. 3 in the idcalized limit of a homogeneous material. In the limit of dilute 


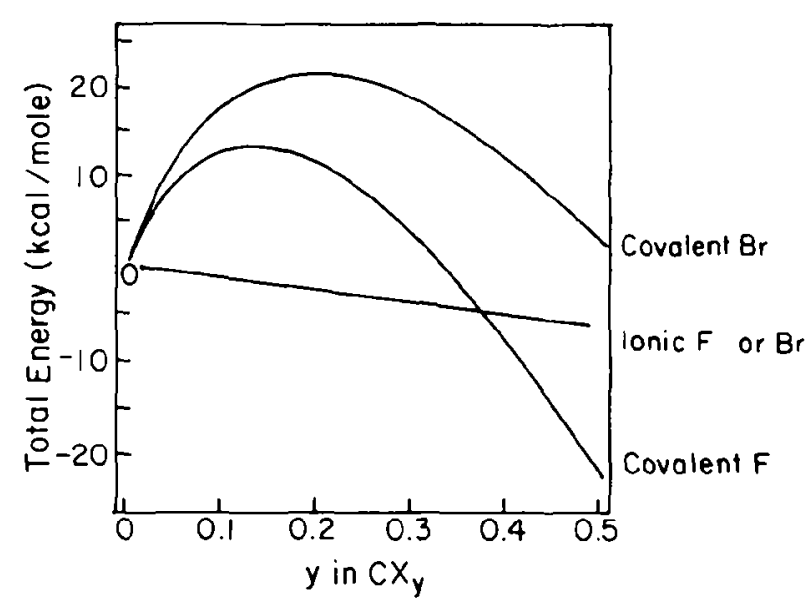

FIG. 3. $A$ schematic of the dependence of the total energy on the fluorine concentration in the two cases of fully ionic bonding and fully covalent bonding, in the two cases of fluorine and bromine intercalated graphite.

halogen concentration, creation of a covalent bond is prohibitively expensive in terms of strain and resonance energy so that only ionic bonds are created. At high fluorine concentrations, covalent bonding of carbon to fluorine is favored because of the strength of the fluorine-carbon covalent bond. In contrast, the brominc-carbon covalent bond is much weaker so that ionic $\mathrm{C}-\mathrm{Br}$ bonding is favored over covalent $\mathrm{C}-\mathrm{Br}$ bonding for all bromine concentrations. Similarly, Fig. 4 shows a plot of the dependence of the total energy as a function of the fraction $f$ of covalent bonds versus ionic bonds. Ionic bromine bonding is favored for all bromine concentrations. However, at high fluorine concentrations, two energy minima exist. These two minima correspond to having all fluorine bonds ionic, or all fluorine bonds

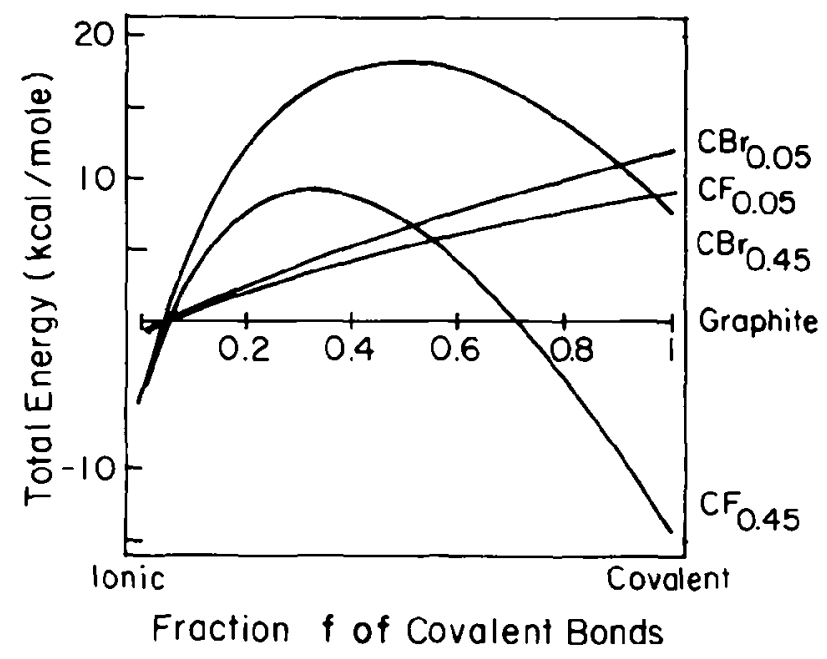

FIG. 4. The dependence of the total energy on the fraction of covalent bonds for various halogen concentrations. covalent, with the covalent minimum more favorable than the ionic minimum, and the two minima separated by an energy barrier. Because the energy curve is concave, the most favorable configuration is to separate the system into dilute and concentrated fluorine islands.

Since creating a dilute concentration of $\mathrm{C}-\mathrm{F}$ covalent bonds is energetically costly, there is an energy barrier to the nucleation of a covalent $\mathrm{C}-\mathrm{F}$ phase inside an ionic $\mathrm{C}-\mathrm{F}$ matrix. Usually nucleation phenomena are modeled as the balance bctween a bulk energy gain from the creation of a new and more stable phase $\Delta G^{19}$ and the surface energy of the interface between two different phases. According to our model, for a given halogen concentration, the bulk cnergy gain is duc to creating strong covalent halogen bonds instcad of weak ionic halogen bonds. Therefore, the bulk free energy gain is

$$
\Delta G=E_{\mathrm{cov}}-E_{\mathrm{iun}}-E_{\mathrm{res}}+3\left(E_{s p^{3}}-E_{s p^{2}}\right)
$$

In Eq. (3), the first two terms correspond to the difference in energy between covalent and ionic bonding, the third term describes the loss of the resonance energy when covalent bonds are formed, and the last term describes the transformation of $s p^{2}$ carbon-carbon bonds into $s p^{3}$ bonds. Similarly, we can describe an interfacial energy that corresponds to $E_{\text {int }}=E_{\text {strain }}+E_{\text {res }}$ for the case of the nucleation of $C_{2} F$ islands inside the ionic matrix.

\section{APPLICATION OF THE MODEL TO FLUORINE AND BROMINE GIC'S}

We now apply our model to explain the observed differences in experimental behaviors between fluorine and bromine GIC's. First of all, let us explain the mixed ionic and covalent nature of fluorine and the ionic nature of bromine. We will assume a homogeneous material. For dilute fluorine concentrations, the creation of covalent bonds is expensive in strain and resonance energy so that bonding is ionic. However, for high fluorine concentrations, the very high bonding encrgy of the fluorine-carbon covalent bond compared to the fluorine-carbon ionic bond makes it energetically favorable to create some covalent bonds. If the fluorine concentration is high enough (maybe beyond about $\mathrm{C}_{3.0} \mathrm{~F}$ ), the energy of the covalent bond can overcome the strain cnergy and the resonance energy. On the other hand, in the case of bromine, because the covalent carbon-bromine bond is not as strong as the $\mathrm{C}-\mathrm{F}$ covalent bond, the ionically bonded bromine GIC is always more stable than the covalently bonded bromine GIC.

Our model also explains the formation of islands ${ }^{4}$ in the covalently bonded fluorine GIC. With respect to our model, the two most stable fluorine concentrations are fully ionic fluorine GIC, which has no covalent bonds, and $\mathrm{C}_{2} \mathrm{~F}$ graphite fluoride, which has only covalent 
bonds. Any intermediate concentration of covalent bonds costs a large strain encrgy. Therefore, it is encrgetically favorable in the fluorine GIC's experimentally investigated to scparate the fluorine concentration into fully ionic dilute islands and fully covalent concentrated islands. In this regard, this model is the same as that of the phase separation of an alloy with a miscibility gap. ${ }^{19}$

Our model does not take into account the presence of defects in the pristine matcrial. Such defects are favorable sites for fluorine covalent bonding (nucleation site), even at low fluorine concentrations. Indecd, a defect is associated with a dangling or strained bond so that formation of a covalent bond does not cost much additional strain or resonance energy. The defects of the pristine material are thus the nucleation sites of our phase transition to a covalently bonded solid. This propensity of defects to bond covalently explains why, for the same intercalated fluorine concentration, less graphitic materials have a more covalent behavior than do highly graphitic materials. This effect is observed in the dependence of the normalized in-plane conductivity $\sigma_{a} / \sigma_{0}$ versus fluorine concentration for graphite CVD fibers as compared to HOPG (graphite with crystallites about $1 \mu \mathrm{m}$ in size), as shown in Fig. 5. In Fig. 5, $\sigma_{0}$ is the conductivity of the pristine material. HOPG is more ordered than CVD fibers, and, therefore, has a smaller density of defects. Nucleation is therefore slower, and for the same fluorine concentration, carbon-fluorine bonding is thus more ionic. As a result, the peak in $\sigma_{a} / \sigma_{0}$ versus [F] is only a factor of 3 for the fibers, whereas it is a factor of 10 for HOPG. The peak is also reached at more dilute fluorine concentrations in CVD fibers than it is in HOPG.

The proposed model also explains the thermal stability of fluorine GIC's upon heating, considering that the ionic bond is not very stable, whereas the covalent bond is extremely stable. (Indeed, highly fluorinated hydrocarbons, such as teflon, are very inert materials.) Upon heating, concentrated fluorine GIC's are found to exhibit a far smaller weight loss than less concentrated materials. ${ }^{11}$ Upon heating, ionic bonds are easily broken so that the weight loss corresponds to the deintercalation of ionically bonded fluorine atoms. Because a majority of carbon-fluorine bonds are covalent in the concentrated fluorine GIC's, these bonds cannot be broken by heating the sample, and the resulting weight loss is thus smaller in the concentrated samples, as compared to dilute fluorine GIC samples.

Finally, we need to explain why fabricating concentrated (beyond $\mathrm{C}_{4.0} \mathrm{~F}$ ) GIC's is so difficult, whereas our model predicts that, once the fluorine concentration becomes high enough, increasing the fluorine concentration should be very easy and disorder should recede to the point where there is no disorder at all in $\mathrm{C}_{2} \mathrm{~F}$.

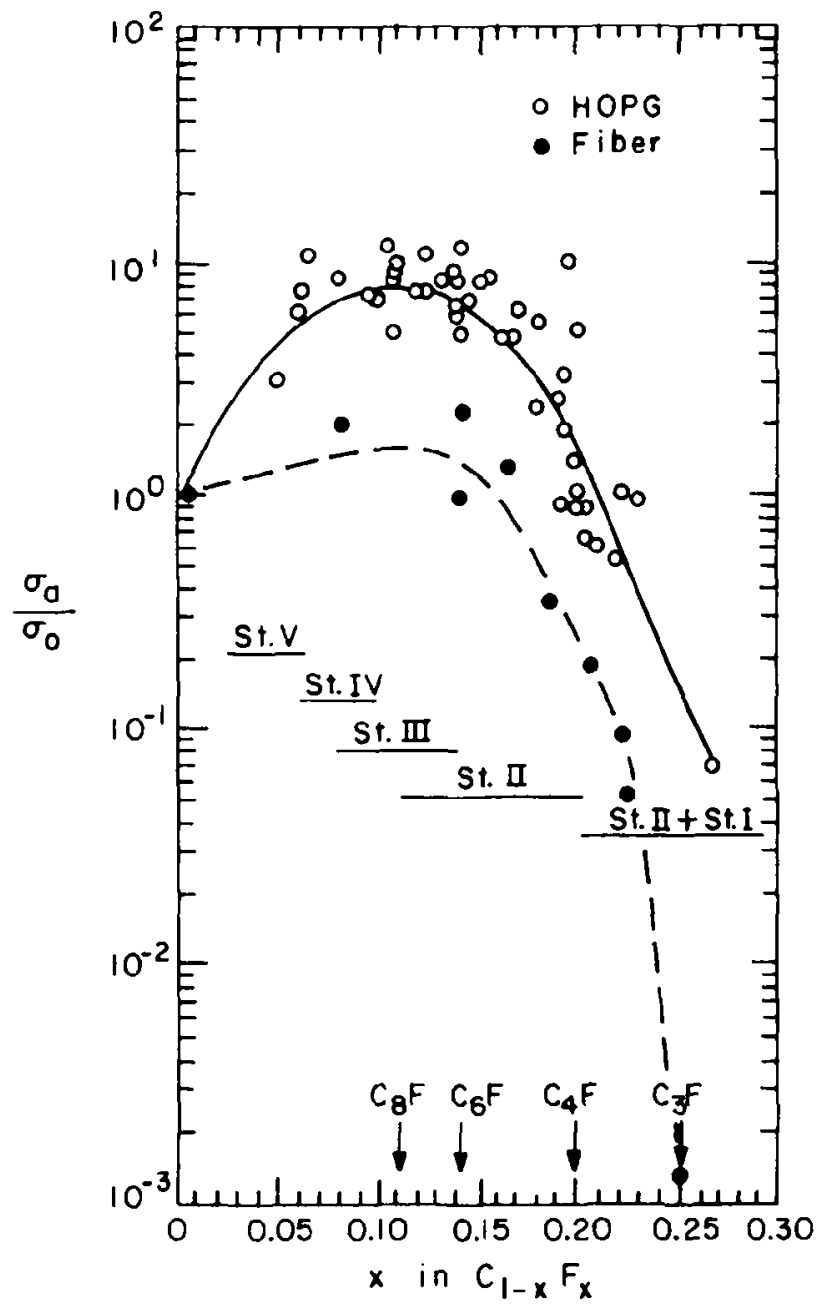

FIG. 5. The dependence on fluorine concentration for the in-plane conductivity $\sigma_{a}$ of fluorine-intercalated HOPG and fluorineintercalated CVD fibers. The data are normalized to the conductivity of the pristine material.

The explanation lies in the microscopic difference between the preparation of GIC's and graphite fluorides. In the case of graphite fluorides, at high temperature, the graphite material gets completely fluorinated to $\mathrm{C}_{2} \mathrm{~F}$, and the reaction front separates the reacted $\mathrm{C}_{2} \mathrm{~F}$ material from the pristine unreacted graphite. On the other hand, in fluorine GIC's, the presence of $\mathrm{AgF}$ allows penetration of the fluorine gas between the graphene layers. Instead of reacting immediatcly with the graphene sheets to form $\mathrm{C}_{2} \mathrm{~F}$, the fluorine atoms actually diffuse and spread all over the available graphite material. As the intercalation proceeds, the fluorine concentration increases all over the samplc. Therefore, when the fluorine concentration becomes high enough, transformation from covalent bonding to ionic bonding occurs in the most concentrated islands, where the nucleation centers are probably the covalent bonds associated with the defects of the pristine 
matcrial. As this transformation procecds, it induces a very high degree of disorder. As discussed above, the main component of this disorder consists of carbon atoms pushed out of the graphene planes. However, the high strain also creates a large density of other defects, mainly stacking faults and dislocations. As the fluorine concentration gets close to the ideal $\mathrm{C}_{2} \mathrm{~F}$ stoichiometry, the strain due to the mixture of $s p^{2}$ and $s p^{3}$ bonds recedes, but the stacking faults and the dislocations do not disappear, and neither do the very disordered regions associated with the boundaries between the covalent grains. We attribute the grain boundaries to the different orientations of the buckling between different islands. The presence of this high degree of disorder in the fluorine GIC's with increasing fluorine concentrations inhibits further fluorine diffusion and the preparation of more concentrated samples.

\section{CONCLUSION}

We have proposed a simple thermodynamic model for the structural and electronic properties of fluorineintercalated graphite. This model explains the striking differences between fluorine GIC's and all other wellstudied GIC's. The model could be tested experimentally and further refined by carrying out in situ measurements as a function of time during fluorine intercalation. It should be possible to observe the real time dependence of the variation of the fraction of ionic and covalent bonds in fluorine GIC's.

Fluorine is found to be a unique intercalate because of the strength of the carbon-fluorine covalent bond and the small size of the fluorine atoms, as opposed to the weakness of the ionic bond. Such a phenomenon is also observed in fluoride intercalated graphite..$^{20}$ However, the case of fluoride GIC's is similar to the case of fluorine GIC's, since, in the fluorides $\mathrm{MF}_{n}$, where $\mathrm{M}$ is a metal, fluorine is the active chemical species that bonds covalently to carbon. In this connection, the other possible candidate of the periodic table for exhibiting a behavior similar to fluorine is oxygen. Indeed, oxygen also forms a very strong bond with carbon and is small enough to enable a dense packing of the oxygen atoms between the graphene planes. Even though no oxygen GIC has been reported in the litcraturc as of now, an oxygen-based covalent layered compound with buckled carbon planes has recently been discovered. ${ }^{21}$ This GIC is the equivalent of the covalent compound $\mathrm{C}_{2} \mathrm{~F}$ in the case of oxygen.

\section{ACKNOWLEDGMENTS}

The authors are grateful to Professor F. Greene, $K$. Kolenbrander, and L. Kimerling from MIT for many enlightening discussions. We also gratefully acknowlcdge support from NSF grant DMR88-19896.

\section{REFERENCES}

1. L. Piraux, V. Bayot, J-P. Issi, M.S. Dresselhaus, M. Endo, and T. Nakajima, Phys. Rev. B 41, 4961 (1990).

2. S. L. di Vittorio, M.S. Dresselhaus, and G. Dresselhaus, in New Horizons in Iow Dimensional Electron Systems-A Festshrift in Honor of Professor H. Kamimura, cdited by H. Aoki, M. Tsukada, M. Schlüter, and F.l. Lévy (Kluwer Academic Publishers, Dordrecht, 1991).

3. S. L. di Vittorio, M.S. Dressclhaus, M. Endo, and T. Nakajima, Phys. Rev. B 43, 12304 (1991).

4. K. Oshida, M. Endo. T. Nakajima, S. L. di Vittorio, M.S. Dresselhaus, and G. Dresselhaus, J. Mater. Res. 8, 512 (1993).

5. S. L. di Vittorio. T. Enoki, M.S. Dresselhaus, G. Dresselhaus, M. Fndo, and T. Nakajima, Phys. Rev. B46, 12723 (1992).

6. I. Ohana, I. Palchan, Y. Yacoby, D. Davidov, and H. Selig. Phys. Rev. B 38, 12627 (1988)

7. M. S. Dresselhaus and G. Dresselhaus, Adv. Phys. 30, 139 (1981).

8. B. T. Kelly, Physics of Graphite (Applied Science, London. 1981).

9. A. Hérold. Proc. Conf. on Intercalation Compounds of Graphite, cdited by F. L. Vogel and A. Hérold, La Napoule, France (1971), p. 1.

10. Landoldt-Börnstein, Numcrical Data and Functional Relationships in Science and Technology. Series III/5a (Springer-Verlag, Berlin, 1983).

11. N. Watanabe, T. Nakajima, and H. Touhara, Graphite Fluorides (Elsevier, Amsterdam, 1988).

12. I. Palchan, D. Davidov, V. Zevin, G. Polatsek, and H. Selig. Synthetic Metals 12, 413 (1985).

13. S. L. di Vittorio, Ph.D. Thesis, Massachusetts Institute of Technology, Department of Materials Science and Engincering (1992).

14. T. Mallouk and N. Bartlett, J. Chem. Soc. Commun. 12, 103 (1985).

15. W. D. Johnson, P. J. Flanders, and J. E. Fischer, Extended $A$ bstracts of the 14th Biennial Conf. on Carbon, Penn State University (1979), p. 296.

16. M.S. Dresselhaus, G. Dresselhaus, K. Sugihara, I. L. Spain, and H. A. Goldberg, Graphite Fibers and Filaments (Springer, New York, 1988).

17. D. D. Ebbing and M.S. Wrighton, General Chemistry (Houghton Mifflin. Boston. MA. 1990).

18. D. D. I. Chung. Phase Transitions 8, 35 (1986).

19. W. D. Kingery, H. K. Bowen, and D. R. Uhlmann, Introduction to Ceramics (Wilcy, New York, 1976).

20. L. Piraux, K. Amine, V. Bayot, J-P. Issi, S. L. di Vittorio, M.S. Dresselhaus, A. Tressaud, S. G. Mayorga, M. Endo, and T. Nakajima, Proc. oth Int. Conf. on Intercalation Compounds, edited by D. Tchouhar and J. Conard, Orléans, France (1991), p. 487.

21. M. Endo, Trans. Jpn. Soc. Elec. Eng. (1988). 